\title{
CONTRIBUTIONS TO THE PROTON GDH SUM RULE ABOVE 2.5 GEV
}

\author{
D.I. SOBER,${ }^{*}$ L. TODOR, ${ }^{\dagger}$ P.E. BOSTED,${ }^{\ddagger}$ S.E. $\mathrm{KUHN}^{\S}$ \\ AND THE CLAS COLLABORATION AT JEFFERSON LAB
}

\begin{abstract}
A measurement of the helicity asymmetry of a substantial fraction of the $\gamma p$ total cross section at photon energies between 2.5 and $5.3 \mathrm{GeV}$ has been performed in Hall B of Jefferson Lab. The preliminary analysis uses only events with at least one charged particle between approximately $8^{\circ}$ and $45^{\circ}$. Assuming that the measured asymmetry applies also to the undetected portion of the cross section, we compare our preliminary result with the Regge parameterization of Bianchi and Thomas and with recent measurements by the GDH Collaboration at Bonn.
\end{abstract}

\section{Introduction}

An experimental test of the Gerasimov-Drell-Hearn (GDH) sum rule ${ }^{1}$ requires measurements of the helicity-dependent cross section difference $\Delta \sigma=\sigma_{1 / 2}-\sigma_{3 / 2}$ over - in principle - the whole range of photon energies above pion production threshold. To date, measurements of the contributions to the GDH sum rule have been made at energies between 0.2 and $0.8 \mathrm{GeV}$ at MAMI (Mainz) ${ }^{2,3}$ and at energies between 0.8 and $3.0 \mathrm{GeV}$ at ELSA (Bonn). ${ }^{3}$ Measurements over portions of the same energy range are planned or in progress at LEGS, GRAAL and Spring-8.

While a large fraction of the sum rule is saturated at low energies, a quantitative test of the sum rule requires measurements at very high energies, and the details of convergence are not well understood. A Regge parameterization of polarized electron scattering data at high energies by Bianchi and Thomas ${ }^{4}$ predicts that a substantial fraction of the sum rule is due to photon energies well above $3 \mathrm{GeV}$. An experiment has been approved at $\mathrm{SLAC}^{5}$ to measure the contribution from photon energies between 5 and $40 \mathrm{GeV}$, but this measurement is still some years in the future.

*The Catholic University of America, Washington, DC 20064

†Carnegie Mellon University, Pittsburgh, PA 15213

$\ddagger$ University of Massachusetts, Amherst, MA 01003

$\S$ Old Dominion University, Norfolk, VA 23529 
With the hope of providing some unique information about the evolution of the GDH sum rule in the energy range 3 to $5 \mathrm{GeV}$, we have performed a measurement of the helicity asymmetry of the $\gamma p$ cross section in a short run in Hall B of Jefferson Lab.

\section{Experiment}

The 3-day tagged-photon run took place in January 2001 during an extended run period devoted to measurements of double-polarization variables in electron scattering, using a dynamically polarized ammonia target. Neither the target nor the beam line were optimized for photon running. In particular, the absence of the "start counter" which normally surrounds the target for photon running meant that the trigger rate was dominated by accidental coincidences between the tagging system and the CLAS detector. In order to make the experiment sensitive to all-neutral events, the trigger required only a coincidence between the tagger and a hit in the forward electromagnetic calorimeter which spans an angle range of approximately $8^{\circ}$ to $45^{\circ}$, closely matching the $50^{\circ}$ forward aperture of the polarized target magnet.

Circularly polarized photons were produced by the bremsstrahlung of longitudinally polarized electrons. The helicity state of the electron beam was reversed 30 times per second and was encoded in the data acquisition. The beam flux in each helicity state was monitored by a synchrotron light monitor located at the final bend of the electron beamline. The electron beam polarization was checked twice during the run with a Møller polarimeter. The circular polarization of the photons was calculated using the formulas of Olsen and Maximon. ${ }^{7}$

The target polarization was monitored continuously by an NMR readout, and its direction was reversed twice during the run. Approximately $10^{8}$ triggers were acquired in each target polarization state, with average target polarizations of $-70 \%$ and $+79 \%$ as measured by NMR. Additional data for background calculation were taken with an empty target cell (helium-filled and truly empty) and a carbon target.

\section{Analysis}

A good hadronic event was defined by a time coincidence between at least one detected particle and a tagged photon. In the preliminary analysis presented here, we required that an event contained at least one chargedparticle track in the drift chambers. Tracks were projected back to the beam 
line, and the small number of events not pointing back to the polarized target cell were rejected. The event time at the target was calculated from the time measured in the "time-of-flight" scintillation counters which follow the chambers, using a particle identification based on energy deposited in the counters. Events were accepted using time windows of 2,6 and 10 ns. The measured asymmetry was independent of this time window, and for the final analysis only the $10 \mathrm{~ns}$ time window was used.

The raw helicity asymmetry was calculated as $A_{\text {raw }}=\frac{N_{a}-N_{p}}{N_{a}+N_{p}}$, where $N_{a}$ and $N_{p}$ are the number of events, corrected for flux and dead time, with the the beam and target spins antiparallel and parallel respectively. The true asymmetry is equal to this ratio divided by the product of photon polarization, target proton polarization and dilution factor.

The asymmetry was calculated from the data both with and without empty-target subtraction. Previous work by the collaboration using this target indicated that subtracting the (helium-filled) empty target data led to smaller systematic uncertainties in the effective target thickness and dilution factor. We obtained nearly identical results by both methods, and only the empty-target-subtracted results are shown here.

The data for each sign of target polarization were analyzed separately. The results were acceptably consistent, with a $\chi^{2}$ of 10.7 for 6 degrees of freedom, and the two target polarizations were combined to give a single result for each energy bin.

From the number of accepted events and our best (relatively crude) estimates of the target thickness and photon flux, we also attempted to calculate the total measured cross section in this experiment. Using the Particle Data Group's parameterization of the total $\gamma p$ cross section, ${ }^{6}$ we estimate that the measured events with charged-particle tracks constitute approximately $50 \%$ of the total cross section. Most of the "missing" cross section is presumably due to the incomplete angular coverage of CLAS as well as the all-neutral events. Analysis of the all-neutral events is still in progress, and may increase this fraction. Since there is no way to extrapolate the effects of the missing part of the cross section on the asymmetry, we simply present the measured asymmetry as a first approximation to the total helicity asymmetry in this energy region.

\section{Results}

The helicity asymmetry $A=\frac{\sigma_{1 / 2}-\sigma_{3 / 2}}{\sigma_{1 / 2}+\sigma_{3 / 2}}$ in each of 6 photon energy bins, averaged over the two target polarizations, is shown in Figure 1. Also shown 


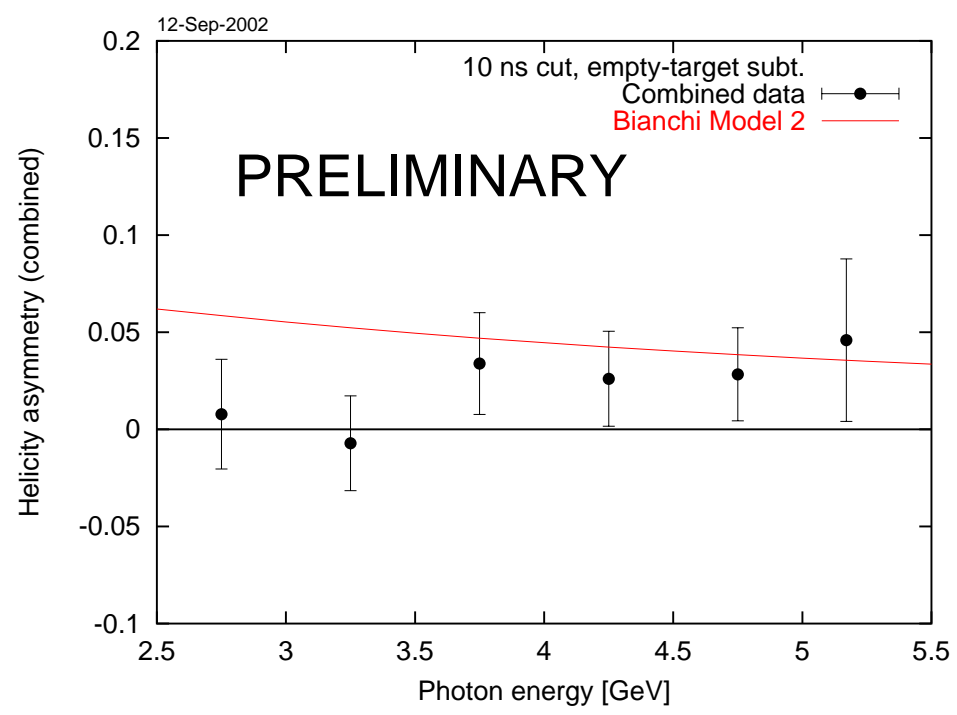

Figure 1. Helicity asymmetry, averaged over the two target polarizations.

is the prediction of the model of Bianchi and Thomas, ${ }^{4}$ converted from cross section difference $\Delta \sigma=\sigma_{1 / 2}-\sigma_{3 / 2}$ to asymmetry $A=\Delta \sigma / 2 \sigma_{\text {tot }}$ using the Particle Data Group ${ }^{6}$ parameterization of the $\gamma p$ total cross section, which fits the world data down to $E_{\gamma}=2.5 \mathrm{GeV}$. It should be noted that the sign convention used here is the opposite of that used in the Mainz and Bonn experiments, ${ }^{2,3}$ so that the positive asymmetry observed here implies a change in the sign of the asymmetry between $800 \mathrm{MeV}$ and our energy region. This is consistent with the recent preliminary results from Bonn. ${ }^{3}$

If we assume that the unmeasured part of the total cross section has the same helicity asymmetry as measured in this experiment, we can estimate the contribution of our energy region to the GDH sum rule. We convert our measured asymmetry to a cross section difference $\Delta \sigma=\sigma_{1 / 2}-\sigma_{3 / 2}$ using the Particle Data Group ${ }^{6}$ parameterization of the $\gamma p$ total cross section. The results are shown in Figure 2, together with the predictions of the three models of Bianchi and Thomas. (Model 2 is their best fit to electron scattering data, while Models 1 and 3 use some limiting assumptions.)

It is seen in Figure 1 that our results fall well below the Bianchi-Thomas parameterization at the low-energy end, but are consistent with it at our highest energies. Since Bianchi and Thomas predict a significant contribution to the GDH sum rule above $3 \mathrm{GeV}$ (approximately $13 \mu \mathrm{b}$, compared to 


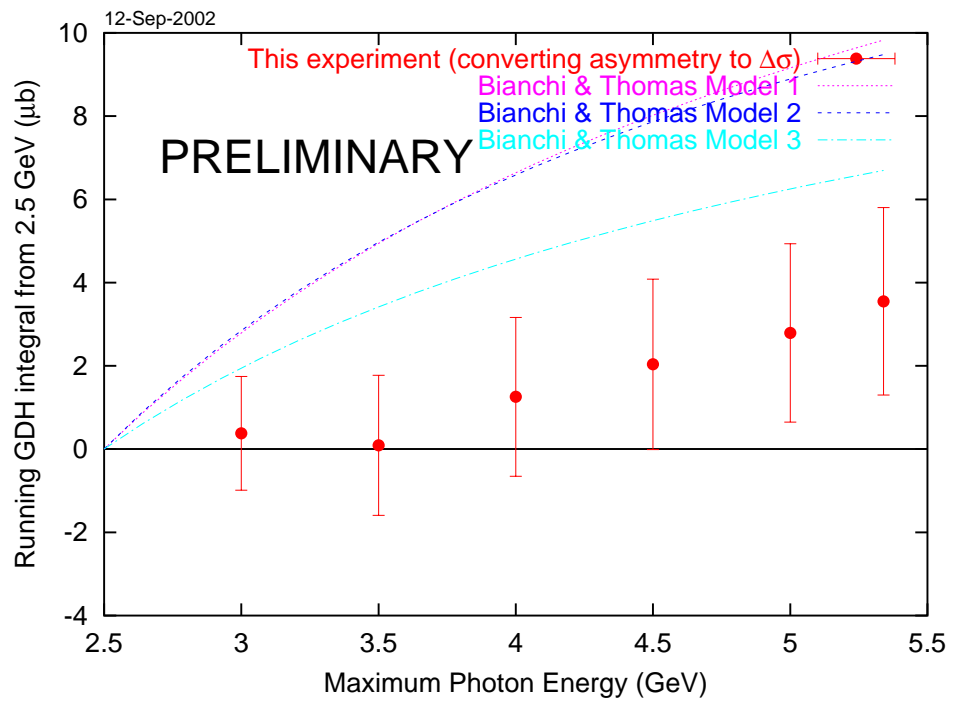

Figure 2. Running GDH integral beginning at $2.5 \mathrm{GeV}$. Data points: this experiment, using Particle Data Group parameterization of the $\gamma p$ total cross section. Curves: Bianchi and Thomas Models 1, 2 and 3.

the sum rule value of $205 \mu \mathrm{b}$ ), it is clear that an investigation of this highenergy region is of great interest. For the energy region between 3 and 6 $\mathrm{GeV}$, a new proposal is being developed for Hall $\mathrm{B}$ at Jefferson Lab which will use the new frozen-spin polarized target which is being constructed for CLAS, and possibly use the forward-angle calorimeter which is being built for an upcoming experiment on deeply virtual Compton scattering ${ }^{8}$ to extend the small-angle acceptance of the CLAS detector.

At higher energies, the planned SLAC experiment ${ }^{5}$ will use a dynamically polarized ammonia target inside a dedicated total-cross-section detector, and will use coherent bremsstrahlung to produce a quasi-monoenergetic photon source at energies between 5 and $40 \mathrm{GeV}$.

\section{Conclusion}

We have measured the helicity asymmetry of an appreciable part of the total $\gamma p$ cross section between 2.5 and $5.3 \mathrm{GeV}$. The measured asymmetries are somewhat lower than the predictions of Bianchi and Thomas, and are qualitatively consistent with the trend of the latest data from Bonn at the lower energies. Additional measurements at these and higher energies, such as those proposed at SLAC, will be important in determining the details of 
the convergence of the GDH sum rule.

\section{Acknowledgments}

This work was supported by the National Science Foundation, the U.S. Department of Energy, the Italian Istituto Nazionale di Fisica Nucleare, the French Commissariat à l'Energie Atomique, and the Korea Science and Engineering Foundation. The Southeastern Universities Research Association (SURA) operates the Thomas Jefferson National Accelerator Facility for the U.S. Department of Energy under contract DE-AC05-84ER40150.

\section{References}

1. S.D. Drell and A.C. Hearn, Phys. Rev. Lett. 16, 908 (1966); S.B. Gerasimov, Sov. J. Nucl. Phys. 2, 430 (1966)

2. J. Ahrens et al., Phys. Rev. Lett. 87, 022003 (2001).

3. P. Pedroni, contribution to this workshop.

4. N. Bianchi and E. Thomas, Phys. Lett. B 450, 439 (1999).

5. SLAC Proposal E-159, "Proposal to Measure $\Delta \sigma^{\gamma n}(k)$ and the High Energy Contribution to the Gerasimov-Drell-Hearn Sum Rule," P. Bosted and D. Crabb, spokespersons (2000).

6. Particle Data Group: D. E. Groom et al., Eur. Phys. J. C 15, 1 (2000).

7. H. Olsen and L.C. Maximon, Phys. Rev. 114, 887 (1959).

8. Jefferson Lab Proposal 01-113, "Deeply Virtual Compton Scattering at 6 GeV with CLAS," V. Burkert, L. Elouadrhiri, M. Garcon and S. Stepanyan, spokespersons (2001). 